\title{
Prevalence of Hypertension and Glycaemic Control in Adult Type-2 Diabetes Patients: A Preliminary Retrospective Cohort Study in Kuantan, Pahang, Malaysia
}

\author{
Fa'iza Abdullah ${ }^{\mathrm{a}}$, Tin Myo Han ${ }^{\mathrm{b}}$, Mohd Basri Mat Nor ${ }^{\mathrm{c}}$, Mohd Aznan MA ${ }^{\mathrm{a}}$, Ida Zuriaty Ismail ${ }^{\mathrm{d}}$ \\ ${ }^{a}$ Department of Family Medicine, Kulliyyah of Medicine, International Islamic University Malaysia, \\ 25200 Kuantan, Pahang \\ ${ }^{b}$ Medical Statistics Unit, Kulliyyah of Dentistry, International Islamic University Malaysia, \\ 25200 Kuantan, Pahang \\ ' Department of Anesthesiology \& Critical Care, Kulliyyah of Medicine, International Islamic University \\ Malaysia, 25200 Kuantan, Pahang \\ ${ }^{d}$ Klinik Kesihatan Bandar Kuantan, Jalan Bukit Sekilau 25000, Bandar Kuantan
}

\begin{abstract}
Introduction: Hypertension (HPT) is the most common co-morbidity among type-2 diabetes mellitus (T2DM) patients which ominously increased their morbidity and mortality from cardiovascular diseases (CVD). We aimed to determine the prevalence and control status of HPT, and also the glycemic control among T2DM patients in a primary care clinic in Kuantan, Pahang. Methods: It was a retrospective study of 154 T2DM patients' records, aged $\geq 18$ years selected by random sampling. The statistical analysis is done by using Chisquare test, paired sample "t" test and ANOVA "F" test. Results: Among T2DM patients; $47 \%$ were Malay, $45 \%$ Chinese and $9 \%$ Indian. The prevalence of HPT was $72.1 \%$ and majority of T2DM patients were women $(60 \%)$. Out of 82 T2DM aged $>60$ years, $80.5 \%$ were hypertensive. $67.2 \%$ of T2DM patients between the age of 40-60 years and $25 \%$ age $<40$ years were also hypertensive $(p=0.003)$. BP-controlled status were classified into controlled, uncontrolled, systolic and diastolic HPT. All patients were compared between the last visit and one year before, which reported $55.8 \%$ versus $33.1 \%, 14.9 \%$ versus $51.9 \%, 20.1 \%$ versus $10.4 \%$ and $9.1 \%$ versus $4.5 \%$ respectively. There were significant rises in percentage of systolic BP (by $9.7 \%$ ) and diastolic HPT (by 4.6\%) $p<0.0001$, from the first visit. BP controlled status for aged group $>60$ years showed increments in systolic HPT and diastolic HPT which were significant $(p<0.0001)$. Regarding glycemic parameters, $71.4 \%$ T2DM patients had poor controlled level of Hb1Ac $(\geq 6.5)$ and only $20.1 \%$ remained controlled after one year $(\mathrm{p}<0.0001)$. Conclusions: This pilot study found high prevalence of HPT, increasing prevalence of systolic HPT and diastolic HPT in older age group as well as poor glycemic control among T2DM patients.
\end{abstract}

KEYWORDS: Controlled blood pressure, systolic hypertension, diastolic hypertension, HbA1C difference, primary care clinic

\section{INTRODUCTION}

Cardiovascular diseases (CVD) which include stroke, heart failure and myocardial infarction accounts for $65 \%$ of the cause of death in type-2 diabetes (T2DM) patients. ${ }^{1}$ Hypertension, which is the most common co-morbidity among T2DM patients, doubles the risk of CVD, thus increased risk of morbidity and mortality. ${ }^{2}$ In Malaysia, the estimated prevalence of

Corresponding Author:

Dr. Fa'iza Abdullah,

Department of Family Medicine,

Kulliyyah of Medicine,

International Islamic University Malaysia,

25200 Kuantan, Pahang, Malaysia

Email:drfaiza@iium.edu.my

Tel: +609570400 Ext : 4584

Fax: +6095716542 hypertension for adults aged 30 years and above was $43.5 \%$ in $2011 .^{3}$ According to Malaysian Clinical Practice Guideline (CPG) 2009 - Management of T2DM (4th Edition), the prevalence of hypertension in T2DM is reported to be around $40-80 \%$ which estimated to be 1.5-3.0 times greater than for nondiabetes person. ${ }^{4-6}$ Therefore hypertension should be detected and treated early in the course of T2DM to prevent macrovascular complications in CVD and to delay the progression of microvascular complications such as renal disease, arterial disease and diabetic retinopathy.

Hypertension may be present during the diagnosis or it may predate the onset of T2DM. It also could develop later due to diabetic nephropathy. ${ }^{2}$ HPT is defined as persistent elevation of systolic blood pressure (BP) $\geq 140 \mathrm{mmHg}$ and/or diastolic $\mathrm{BP} \geq 90$ $\mathrm{mmHg}$ for adult $\geq 18$ years of age. ${ }^{3}$ However, there 
is no absolute agreement in setting a BP diagnostic level for HPT in diabetes patients. Due to high CVD risk, these thresholds are reduced to SBP > 130 $\mathrm{mmHg}$ and /or DBP > $80 \mathrm{mmHg}$ for individuals with diabetes to be diagnosed with HPT by many scientific society. ${ }^{7-10}$ National Institute for Health and Clinical Excellent (NICE) maintain the threshold at the same values as for normal population. ${ }^{11}$

Optimal BP Control is an important approach in reducing the CVD risks. Many previous studies have demonstrated the importance of BP control in diabetes patients. The U.K. Prospective Diabetes study (UKPDS) showed irrefutably that the complications of T2DM could be reduced by improving blood glucose and/or BP control. ${ }^{12}$ The Hypertension Optimal Treatment (HOT) study by Hanson et al helped to establish target diastolic blood pressure (DBP) level in treatment of hypertension. It showed $51 \%$ reduction in major cardiovascular events in diabetes patients with target group DBP $\leq 80 \mathrm{mmHg}$ compared with target group DBP $\leq 90 \mathrm{mmHg}(p=0.005) .{ }^{13}$ In general, the SBP should be targeted to $\leq 130 \mathrm{mmHg}$ and diastolic pressure $\leq 80 \mathrm{mmHg}^{14}$ and this comply with the Malaysian CPG Management of T2DM.

It is imperative to analyze our own primary care clinic (PCC) statistic and the changes of BP control status groups between the last visit (Time-2) and one year before (Time-1) among T2DM patients in order to obtain comprehensive statistical results to comprehend the impact of the disease to the community and government. We aimed to determine the prevalence and control status of hypertension among Type-2 DM patients in outpatients at a major urban primary care clinic in Kuantan; and also to analyze the relationship between hypertension control status and their glycemic control.

\section{MATERIALS AND METHODS}

A retrospective study among 154 records of T2DM patients was conducted at a selected public primary care clinic (PCC) in Kuantan, in 2014. The inclusion criteria pertaining to records selection were T2DM patients who were more than 18 years and those who were on active follow-up (at least two times) within one year from the date of last visit. Exclusion criteria are the records of the patients who were defaulter, illegible handwriting and referred from any tertiary center. A structured data collection format was applied to scrutinize the demographic and clinical background of both hypertension and diabetes. These included the diagnosed year and duration of the disease. We also collected data from the patients' records on BP control status (BP values) and glycemic control status (HbA1c results) at two time points i.e at the last visit (Time-2) and one year before the last visit (Time-1). BP measurements were done by a diabetic educator nurse in the screening area before doctor consultation session using standardized automatic
Omron BP set. Blood for HbA1c was taken by medical assistance (MA) and calculated using HbA1C machine Model D-10 from company BIO-RAD in its own lab. BP control status was classified into controlled $(\leq 130 / \leq 80)$, uncontrolled $(>130 />80)$, systolic hypertension $(>130 / \leq 80)$ and diastolic uncontrolled $(\leq 130 />80)$ for individual with T2DM ${ }^{2}$. HbA1C (\%) $<6.5$ is defined as good glycemic control and if the level is equal to or more than ( $\geq$ ) $6.5 \%$ was defined as uncontrolled glycemic status. Prevalence of hypertension was computed and its relationship to demographic background was analyzed by using an appropriate cross-analysis test such as Pearson chi-square, Yates correction and fisher's exact test. BP controlled status in accordance with age and glycemic control status were also analyzed using chi-square tests. A cross analysis on blood pressure control status at Time-1 and Time-2 was also done. Sample size for the study was calculated using "Stata IC-12 software" based on total estimated of DM patients' records of the clinic under study and prevalence of hypertension in the DM patients. A total 156 T2DM records were selected from the total number of 1280 T2DM patients registry by applying systematic randomized sampling method. First, sampling interval (k) was determined by the total number of registered T2DM records divided by the total number of the sample. Therefore, sampling interval $(k)$ was 8 as it was calculated as 1280 (total number of records) divided by 154 samples. In this study, the first patient's record selected by using random digit table was the seventh one.

Then, the subsequent patient's record was selected at previously determined sampling interval $(\mathrm{k}=8)$. A pilot study on 15 T2DM records at the study site was carried out to look for the feasibility of the data collection process and modification of the data collection tool. Prior to commencement of the study, ethical approval was obtained from local Medical Research Ethics Committee (MREC) and this study was registered under the National Medical Research Register (NMRR-13-847-16398).

\section{RESULTS}

The demographic background and hypertension status of T2DM patients are presented in Table 1. From the total of 154 T2DM patients' record analyzed, the prevalence of HPT in T2DM patients in this study was $72.1 \%$, of whom, the majority were women $(60 \%)$. High prevalence of hypertension was seen among T2DM patients $>60$ years group (80.5\%), followed by $67.2 \%$ in $40-60$ years group and $25 \%$ in patients aged $<40$ years $(p=0.003)$. The ethnic proportions of patients' were 47\% Malay, $44 \%$ Chinese and $9 \%$ Indian. We observed high number of female hypertensive in dependents/housewife group i.e. $76.7 \%(33 / 43)$, but this was not statistically significant. A prevalence of hypertension is significantly higher in Chinese $(82.1 \%)$, but it was almost the same in between Malay (65.3\%) and Indian (64.3\%), $\mathrm{p}=0.032$. 
Table 1: Demographic background of T2DM patients

\begin{tabular}{|c|c|c|c|c|}
\hline \multirow[t]{2}{*}{ Variables } & \multicolumn{2}{|c|}{ Hypertension } & \multirow{2}{*}{$\begin{array}{l}\text { Total } \\
(\mathrm{n}=154)\end{array}$} & \multirow[t]{2}{*}{$\mathrm{p}$} \\
\hline & $\begin{array}{c}\text { Yes } \\
(n=111)\end{array}$ & $\begin{array}{c}\text { No } \\
(n=43)\end{array}$ & & \\
\hline \multicolumn{5}{|l|}{ Age Group years } \\
\hline$\leq 40$ & $2(25)$ & $6(75)$ & $8(5.2)$ & 0.003 \\
\hline $40-60$ & $43(67.2)$ & $21(32.8)$ & $64(41.6)$ & \\
\hline$\geq 60$ & $66(80.5)$ & $16(19.5)$ & $82(53.2)$ & \\
\hline Gender (male) & $44(67.7)$ & $21(32.3)$ & $65(42.2)$ & 0.300 \\
\hline \multicolumn{5}{|l|}{ Race } \\
\hline Malay & $47(65.3)$ & $25(34.7)$ & $72(46.8)$ & 0.032 \\
\hline Chinese & $55(82.1)$ & $12(17.9)$ & 67(43.5) & \\
\hline Indian & $9(64.3)$ & $5(35.7)$ & $14(9.1)$ & \\
\hline Others & $0(0)$ & $1(100)$ & $1(0.6)$ & \\
\hline \multicolumn{5}{|l|}{ Occupation } \\
\hline Government Staff & $6(50)$ & $6(50)$ & $12(7.8)$ & 0.133 \\
\hline Private Staff & $9(69.2)$ & $4(30.8)$ & $13(8.4)$ & \\
\hline Own business & $3(75)$ & $1(25)$ & $4(2.6)$ & \\
\hline Dependent/Housewife & $33(76.7)$ & $10(23.3)$ & $43(27.9)$ & \\
\hline Retired & $3(50)$ & $3(50)$ & $6(3.9)$ & \\
\hline Other & $0(0)$ & $2(100)$ & $2(1.3)$ & \\
\hline Unknown* & $57(77)$ & $17(23$ & $74(48.1)$ & \\
\hline
\end{tabular}

Data expressed as $\mathrm{n}$ (\%). Comparisons between groups were analyzed by Pearson chi-square test for gender and Fisher's exact test for the rest of categorical variables. Unknown* patients were not included in statistical analysis for occupation category. T2DM: Type-2 diabetes mellitus.

The duration of T2DM and hypertension in relation to year of diagnosis is shown in Table 2. Majority of these patients had duration of diabetes and hypertension for 5-10 years. When we observed the pattern of HPT in relation to year of T2DM diagnosis, mostly was diagnosed earlier (42.3\%). The rests of patients were diagnosed either at the same year $(27.9 \%)$ or after $(29.8 \%)$.

Table 2: Duration of T2DM and Hypertension in relation to year of diagnosis

\begin{tabular}{lccc}
\hline & Frequency & Percentage & Valid percentage \\
\hline Duration of diabetes (years) & & & \\
Less than 5 & $(\mathrm{n}=154)$ & $(\mathrm{n}=154)$ & $(\mathrm{n}=152)$ \\
$5-10$ & 26 & 16.9 & 17.2 \\
$11-15$ & 68 & 44.2 & 45.0 \\
More than 15 & 39 & 25.3 & 25.8 \\
Unknown* & 18 & 11.7 & - \\
& 3 & 1.9 & \\
Duration of hypertension (years) & & & $(\mathrm{n}=106)$ \\
Less than 5 & & & 9.4 \\
5-10 & $(\mathrm{n}=111)$ & $(\mathrm{n}=111)$ & 53.8 \\
11-15 & 10 & 6.5 & 21.7 \\
More than 15 & 57 & 37.0 & 15.1 \\
Unknown* & 23 & 14.9 & - \\
& 16 & 10.4 & $(\mathrm{n}=104)$ \\
Relative time of diagnosis & 5 & 3.2 & 2.9 \\
Both diagnosed at same year & & & 42.3 \\
Hypertension before diabetes & $(\mathrm{n}=111)$ & $(\mathrm{n}=111)$ & 29.8 \\
Hypertension after diabetes & 29 & 28.6 & - \\
Unknown* & 44 & 18.8 & 20.1 \\
\end{tabular}

In valid percentage, the unknown* data were excluded in the analysis (denominator). T2DM=type-2 diabetes mellitus. 
Table 3 shows the changes of BP control status between Time-1 visit (at one year before the last visit) and Time- 2 (at the last visit). BP control status groups were compared between the two visits which were within one year interval. BP controlled status were classified into controlled, uncontrolled, systolic hypertension and diastolic hypertension. Using the sequence of the BP control status mentioned earlier, Time-2 as compared to Time-1, the study findings were $55.8 \%$ vs. $33.1 \%$ for controlled, $14.9 \%$ vs. $51.9 \%$ for uncontrolled, $20.1 \%$ vs. $10.4 \%$ for systolic hypertension and $9.1 \%$ vs. $4.5 \%$ for diastolic hypertension groups. Although more than half T2DM patients had optimize BP control than one year before, there was a significant increase of uncontrolled systolic HPT by $9.7 \%$ and uncontrolled diastolic HPT by $4.6 \%, p<0.0001$.

Table 3: Changes of BP control status between Time-1 and Time-2 visits in one year interval

\begin{tabular}{|c|c|c|c|c|c|}
\hline \multirow[t]{2}{*}{ Time-1 } & \multicolumn{5}{|c|}{ Time-2 } \\
\hline & $\begin{array}{l}\text { Controlled } \\
(\leq 130 / 80)\end{array}$ & $\begin{array}{l}\text { Uncontrolled } \\
(>130 />80)\end{array}$ & $\begin{array}{c}\text { Systolic HPT } \\
(>130 / \leq 80)\end{array}$ & $\begin{array}{c}\text { Diastolic HPT } \\
(\leq 130 />80)\end{array}$ & $\begin{array}{c}\text { Total } \\
\mathrm{n}(\%) \text { at } \\
\text { time } 1\end{array}$ \\
\hline Controlled & 51 & 0 & 0 & 0 & $51(33.1)$ \\
\hline Uncontrolled & 31 & 23 & 19 & 7 & $80(51.9)$ \\
\hline Systolic HPT & 4 & 0 & 12 & 0 & $16(10.4)$ \\
\hline Diastolic HPT & 0 & 0 & 0 & 7 & $7(4.5)$ \\
\hline $\begin{array}{l}\text { (Total } n(\%) \text { at } \\
\text { Time-2) }\end{array}$ & $86(55.8)$ & $23(14.9)$ & $31(20.1)$ & $14(9.1)$ & 154 \\
\hline$p$ value & 's exact te & $p=<0.0001$ & & & \\
\hline
\end{tabular}

Comparisons were analyzed using fisher's exact test. Time- 1 visit: at one year before the last visit. Time- 2 visit: at the last visit. T2DM: type-2 diabetes mellitus.

A statistically significant improvements of BP control were observed in patients age group between 40-60 years and $\geq 60$ years, $p$ values < 0.0001 and $p<0.0001$ respectively. However, there was slight increment of number of patients in systolic HPT group and diastolic HPT group in both aged categories. The changes of BP control status at two different time visits with different age groups are shown in Table 4. Highest raised in percentage was observed in the systolic HPT group for age $\geq 60$ years from $69 \%$ to $71 \%$ after 1 year and this increment is alarming.
Relationship between BP controlled status and glycaemic control of T2DM patients as reflected by changes of HbA1C level among different BP controlled status at the two time visits interval is illustrated by Figure 1. Uncontrolled HbA1c level $(\geq 6.5 \%)$ in the controlled BP $(\leq 130 / 80)$ and uncontrolled BP $(>130 / 80)$ groups decreased after one year but increased for systolic and diastolic HPT groups though the changes were no significant different.

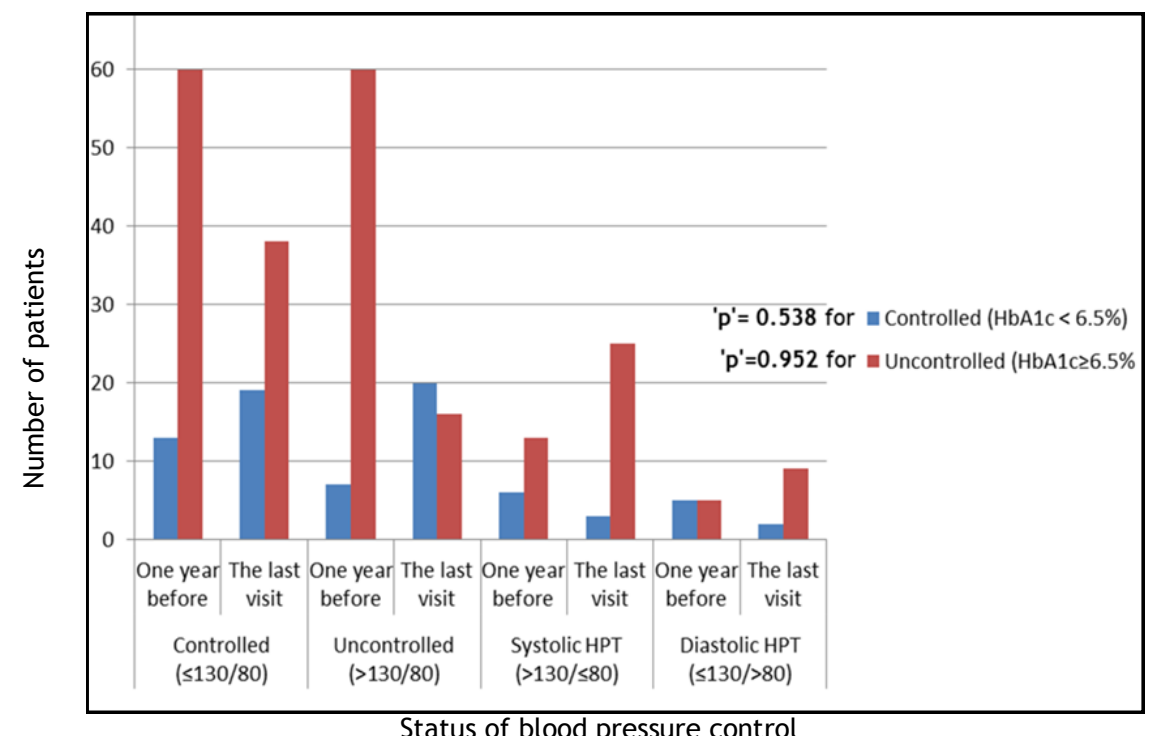

Figure 1: Relationship in between blood pressure control status and glycaemic control of T2DM Patients 
Figure 2 demonstrated that majority of T2DM patients had HbA1c level $\geq 6.5 \%$ for both time visits i.e. at one year before and at the last visit which were $75.3 \%$ and $76.0 \%$ respectively. $71.4 \%(110 / 154)$ T2DM patients remained uncontrolled after one year follow up and only $20.1 \%$ (31/154) remained controlled after one year. This figure was alarming as it significantly $(p<0.0001)$ indicated that majority of T2DM patients have not been within good glycemic control after one year follow up.

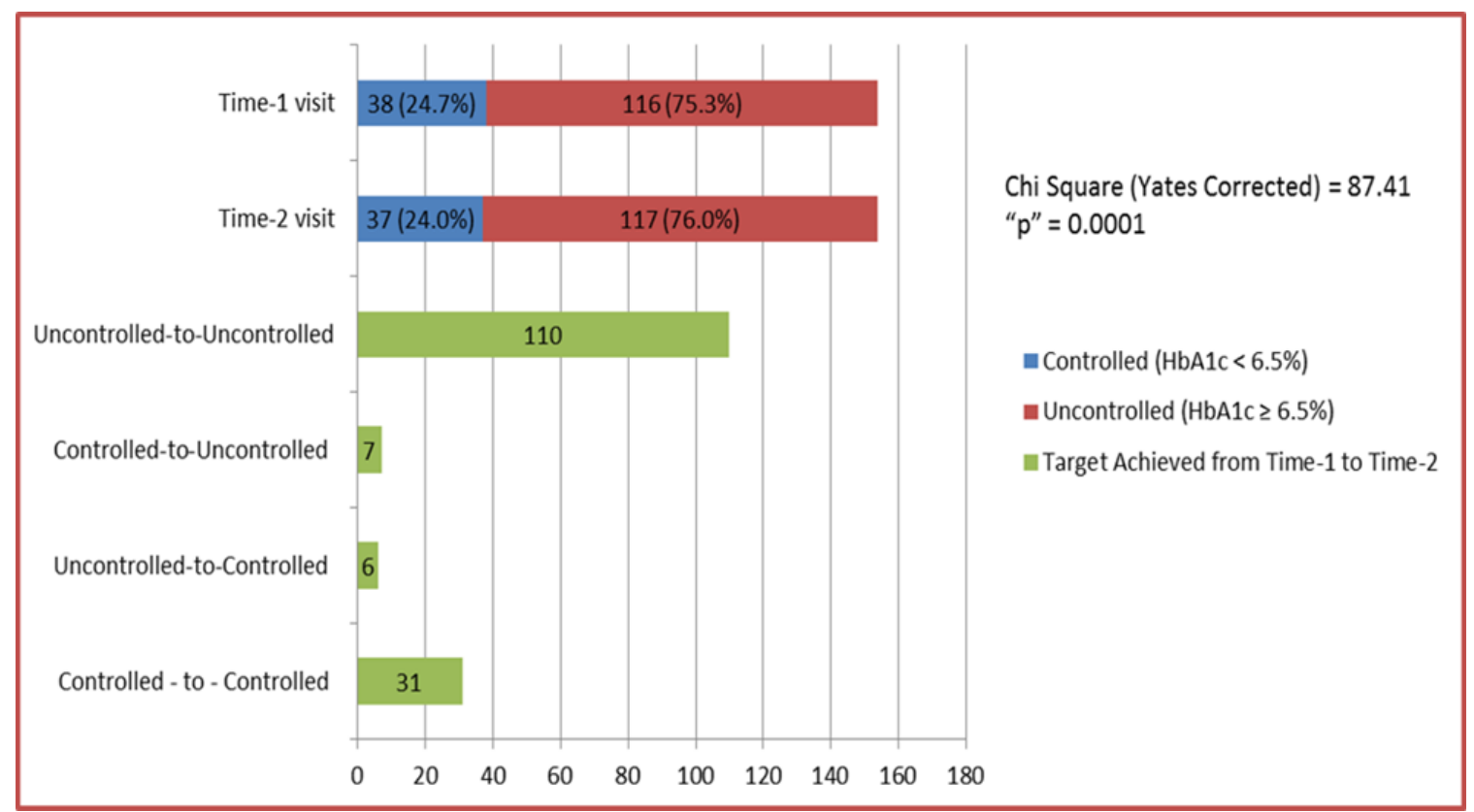

Figure-2: Changes of glycemic control status (HbA1C) between Time-1 visit and Time-2 visit in T2DM patients

\section{DISCUSSION}

In this retrospective study, we showed that more than two third of T2DM patients treated in our public primary care clinic sufferred from hypertension (72.1\%). About $70 \%$ developed hypertension at the same year or predate the onset of hyperglycaemia. Although more than half of them had optimized BP control than one year before, there was a significant increase of uncontrolled systolic HPT and uncontrolled diastolic HPT. 71.4\% of T2DM patients had poor glycaemic control after one year follow up and only $20.1 \%$ remained controlled as reflected by $\mathrm{HbA} 1 \mathrm{c}<6.5$. Our prevalence was comparable to Malaysian CPG 4th Edition-Management of T2DM and National Diabetic Registry 2013 which reported $40 \%-80 \%$ and $72.68 \%$ respectively.

Two recent local studies by Mafauzy et al and Ibrahim et al reported higher prevalence of hypertension in T2DM of $80.3 \%{ }^{15}$ and $92.7 \%{ }^{16}$. Substantial number of our T2DM patients had suboptimal BP and glycaemic control and this may predisposed them to increased risk of morbidity and mortality from cardiovascular events. Further health promotion and prevention activities such as therapeutic lifestyle changes, screening for diabetic complications, diabetic counseling and appropriate treatment should be carried out to both T2DM and hypertensive patients at primary care level in order to reduce its micro- and macrovascular complications.
The proportion of ethnic groups in this study i.e. Malay $(47 \%)$, Chinese $(44 \%)$ and Indian (9\%) who had diabetes mellitus generally is not correspondent to the Pahang state ethnicity distribution of $70 \%, 20 \%$ and $4 \%$ respectively. This study was carried out at the clinic situated in the urban area which has high density of Chinese population coming for treatment. This study also shows a significantly higher percentage of Chinese with T2DM who had concomitant hypertension in comparison to other races. However, lack of literature review regarding this 'higher' prevalence of hypertension in Chinese T2DM suggesting of further research should be carried out in future. The high prevalence of hypertension in T2DM was observed in age group more than 60 years old. This result was anticipated, because the prevalence of hypertension in general population for older people reported in previous studies were high e.g. in Malaysia $74.0 \%{ }^{17}$ and in Singapore $73.9 \%{ }^{18}$.

In this study, most of T2DM patients were having diabetes for $1-10$ years duration and of this, $60 \%$ was presented with hypertension for similar duration. Nearly half of patients developed hypertension earlier than diabetic, one fourth diagnosed in the same year and one fourth after. This demonstrates that majority of hypertension were already present in the year diabetes was diagnosed. Type-2 diabetes usually being diagnosed at advanced age or with obesity, both of which carry increase risk of elevated BP. In these 
conditions, it makes difficult to accredit that hypertension is solely due to diabetes ${ }^{19}$. Age at onset, duration of diabetes and presence of hypertension has emerged as independent and significant predictors of complications in other studies in Malaysia. ${ }^{15}$

T2DM patients in this study had an improvement on BP control after 1 year follow up. The increment in percentage of good BP controlled group was $22.7 \%$ (from $33.1 \%$ to $55.8 \%$ ) and reduction of uncontrolled BP group was $38.0 \%$ (from $52.9 \%$ to $14.9 \%$ ) after 1 year. As aforementioned, many scientific societies reduced BP diagnostic level for HPT in T2DM patients to SBP $\geq 130$ and /or DBP $\geq 80$ owing to high CVD risk. ${ }^{7-10}$ Hence in this study, there was a significant increase of systolic HPT (increment of 9.7\%) and diastolic HPT (increment of 4.6\%) after 1 year which is alarming as it carries with it a poor prognosis. $^{3}$ We reported a significantly higher prevalence of systolic HPT among T2DM in older people (> 60 years old) after one year (69\%-to-71\%). The prevalence of systolic HPT increases with age, and in population aged $>50$ years, it represents the most common form of hypertension. ${ }^{3}$ There is a consistence increased risk for micro- and macrovascular diseases with elevated systolic BP, ${ }^{20}$ thus controlling the systolic BP is obligatory to reduce complications. A slow reduction of systolic pressure in older patients is important and a target level of systolic BP adjusted around $140 \mathrm{mmHg}$ seems desirable. ${ }^{21}$

Although in both $>60$ years and 40-60 years age groups showed an increased in diastolic HPT (by number of patients) after one year, the increment was observed highest in the latter(57\%). Diastolic HPT usually predominates before age 50 years, either alone or in combination with systolic HPT. ${ }^{3}$ Diastolic HPT is more potent cardiovascular risk predictor than SBP until age 50; thereafter, SBP become more important. ${ }^{22}$ Further studies in T2DM patients with systolic hypertension and diastolic hypertension should be carried out to demonstrate the beneficial effect of the treatment in both groups, and in the treatment of hypertension among the older age group in general.

Although uncontrolled $\mathrm{HbA} 1 \mathrm{C}$ level $(\geq 6.5 \%)$ in the controlled BP $(\leq 130 / 80)$ and uncontrolled BP $(>130 / 80)$ groups decreased after one year, it was not significant. The percentage decreased was small and uncontrolled $\mathrm{HbA} 1 \mathrm{c}$ levels noted to rise in the systolic HPT and diastolic HPT groups after one year. As perceived in Figure-2, it showed that majority of T2DM patients (more than 75\%) had not been within good glycemic control $(\mathrm{HbA} 1 \mathrm{c} \geq 6.5 \%)$ on both visits. This significant finding of uncontrolled HbA1c status was comparable with a recent study by Ismail et al. in 2011. They concluded that there was a poor glycaemic control among T2DM studied in $72.9 \%$ and $75.3 \%$ of both male and female patients' respectively. ${ }^{23}$ National Diabetes Registry 2009-2012 revealed the percentage of patients reaching clinical targets for HbA1c in 2012 was only 23.8\%. Results of another one local study, DiabCare 2008 reported just $11.4 \%$ of their T2DM patient cohorts achieving glycemic target. This study also showed that majority of these patients had poor adherence to diet, exercise and self-testing blood glucose which ominously contribute to the poor glycemic control $^{15}$. There is a need of more aggressive and effective methods to educate both patients and health personnel on importance of achieving good glycemic control.

\section{CONCLUSION}

The results of our study revealed that there was a high prevalence of HPT among T2DM patients. Hypertension present earlier or in the same year when T2DM was diagnosed in most of the patients. Higher percentages of systolic HPT and diastolic HPT were seen in T2DM age $>60$ years old.

Majority of T2DM patients had poor glycemic control. There is no significant difference in the glycemic control status after one year followed up in both controlled-BP and uncontrolled-BP groups.

\section{CONFLICT OF INTEREST - None}

\section{REFERENCES}

1. Webcasts JN. Executive summary of the third report of the National Cholesterol Education Program (NCEP) expert panel on detection, evaluation, and treatment of high blood cholesterol in adults (adult treatment panel III). Jama, 2001; 285:2486-97.

2. Salanitro $A H$ and Roumie CL. Blood pressure management in patients with diabetes. Clinical Diabetes, 2010; 28:107-14.

3. Malaysian Society of hypertension M, Clinical Practice Guidelines: Management of Hypertension (4th Edition). 2013, Malaysia.

4. Mohamed M. An audit on diabetes management in Asian patients treated by specialists: the Diabcare-Asia 1998 and 2003 studies. Current Medical Research and Opinion $®, 2008 ; 24: 507$ 14.

5. Turner R, Holman R, Matthews D, et al. Hypertension in diabetes study (Hds). 1. Prevalence of hypertension in newly presenting Type-2 diabetic-patients and the association with risk-factors for cardiovascular and diabetic complications. J HYPERTENS, 1993; 11:309-17.

6. Rampal L, Rampal S, Azhar M, and Rahman AR. Prevalence, awareness, treatment and control of hypertension in Malaysia: a national study of 16,440 subjects. Public health, 2008; 122:11-8.

7. American Diabetes Association Treatment of hypertension in adults withdiabetes (Position Statement). 2003. 26 S80-S2.

8. Chobanian AV, Bakris GL, Black HR, et al. Seventh report of the joint national committee on prevention, detection, evaluation, and 
treatment of high blood pressure. Hypertension, 2003; 42:1206-52.

9. Mancia G, De Backer G, Dominiczak A, et al. The task force for the management of arterial hypertension of the European Society of Cardiology. 2007 Guidelines for the management of arterial hypertension: The Task Force for the Management of Arterial Hypertension of the European Society of Hypertension (ESH) and of the European Society of Cardiology (ESC). Eur Heart J, 2007; 28:1462536.

10. Harper W, Clement M, and Goldenberg R. Canadian Diabetes Association 2013 Clinical Practice Guidelines for the Prevention and Management of Diabetes in Canada: pharmacologic management of type 2 diabetes. Can J Diabetes, 2013; 37:S61-S8.

11. Conditions NCCfC, Type 2 diabetes: national clinical guideline for management in primary and secondary care (update). 2008: Royal College of Physicians (UK).

12. Group UPDS. Tight blood pressure control and risk of macrovascular and microvascular complications in type 2 diabetes: UKPDS 38. BMJ: British Medical Journal, 1998; 317:703.

13. Hansson L, Zanchetti A, Carruthers SG, et al. Effects of intensive blood-pressure lowering and low-dose aspirin in patients with hypertension: principal results of the Hypertension Optimal Treatment (HOT) randomised trial. The Lancet, 1998; 351:1755-62.

14. Tjoa HI and Kaplan NM. Nonpharmacological treatment of hypertension in diabetes mellitus. Diabetes care, 1991; 14:449-60.

15. Mafauzy M, Hussein Z, and Chan S. The status of diabetes control in Malaysia: results of DiabCare 2008. Med J Malaysia, 2011; 66:175-81.

16. Salwa Selim Ibrahim ASIB, Fita Rahmawati, Mohamed Azmi Hassali, and Syed Azhar Syed Sulaiman,, Prevalence And Control of Hypertension Among labetes Patients In Hospital Universiti Sains Malaysia, Malaysia., in Majalah Farmasi Indonesia, . 2010: Indonesia. p. 121 - 8.

17. Kiau B, Kau J, Nainu B, et al. Prevalence, awareness, treatment and control of Hypertension among the elderly: the 2006 National Health and Morbidity Survey III in Malaysia. The Medical journal of Malaysia, 2013; 68:332-7.

18. Malhotra R, Chan A, Malhotra C, and Ostbye T. Prevalence, awareness, treatment and control of hypertension in the elderly population of Singapore. Hypertens Res, 2010; 33:1223-31.

19. Arauz-Pacheco C PM, Raskin P. The treatment of hypertension in adult patients with diabetes. . Diabetes care, 2002; 25:134-47.

20. Adler Al, Stratton IM, Neil HAW, et al. Association of systolic blood pressure with macrovascular and microvascular complications of type 2 diabetes (UKPDS 36): prospective observational study. Bmj, 2000; 321:412-9.
21. van Zwieten PA. Drug treatment of isolated systolic hypertension. Nephrology dialysis transplantation, 2001; 16:1095-7.

22. Franklin SS, Larson MG, Khan SA, et al. Does the relation of blood pressure to coronary heart disease risk change with aging? The Framingham Heart Study. Circulation, 2001; 103:1245-9.

23. Ismail $H$, Hanafiah $M$, Saa'diah $S$, and Yunus $M$. Control Of Glycosylated Haemoglobin (Hba1c) Among Type 2diabetes Mellitus Patients Attending An Urban Health Clinic In Malaysia. Medical And Health Science Journal, 2011; 9:5865. 
